\section{Histoire Épistémologie Langage}

43-2 | 2021

Linguistique psychologique et sémiotique : le contexte allemand et son influence

\title{
Le béhaviorisme sémiotique de Jakob von Uexküll
}

\section{Didier Samain}

\section{(2) OpenEdition}

\section{Journals}

Édition électronique

URL : https://journals.openedition.org/hel/1209

DOI : 10.4000/hel.1209

ISSN : 1638-1580

Éditeur

Société d'histoire et d'épistémologie des sciences du langage

\section{Édition imprimée}

Date de publication : 31 décembre 2021

Pagination : 113-136

ISBN : 9791091587150

ISSN : 0750-8069

Référence électronique

Didier Samain, "Le béhaviorisme sémiotique de Jakob von Uexküll », Histoire Épistémologie Langage [En ligne], 43-2 | 2021, mis en ligne le 01 décembre 2021, consulté le 17 décembre 2021. URL : http:// journals.openedition.org/hel/1209; DOl : https://doi.org/10.4000/hel.1209

\section{(c) (i) (9)}

HEL is licensed under a Creative Commons Attribution-NonCommercial-NoDerivatives 4.0 International License 


\section{LE BÉHAVIORISME SÉMIOTIQUE DE JAKOB VON UEXKÜLL}

\section{Didier Samain}

Sorbonne Université, Histoire des théories linguistiques, Paris, France

\begin{abstract}
Résumé - L'article propose une contextualisation des thèses du biologiste balte Jakob von Uexküll (1864-1944), dont la lecture révèle des convergences tout à la fois avec les versions non physicalistes du béhaviorisme et avec le néovitalisme. L'importance de la référence au kantisme conduit par ailleurs à relativiser la thèse d'une discontinuité entre les premiers travaux consacrés aux invertébrés et l'œuvre théorique ultérieure, centrée sur la notion d'Umwelt et sur une réinterprétation "sémiotique» de la biologie. Il apparaît en revanche que cette sémiotique, et les notions associées de signe (Zeichen), de signification (Bedeutung), de caractéristique (Merkmal) et de représentant (Repräsentant), ne correspondent pas à un programme structuré. Dans un contexte historique où la biologie naissante peinait à définir sa spécificité entre le mécanisme incarné par Loeb et la persistance du vitalisme, elle permettait surtout de modéliser pour un coût explicatif réduit les spécificités du vivant.
\end{abstract}

Mots-clés - béhaviorisme, biosémiotique, concepts-frontières, néokantisme, sémiotique, Uexküll, Umwelt, vitalisme
Abstract - The article offers a contextualization of the theories of the Baltic biologist Jakob von Uexküll (1864-1944). Uexküll's works show convergence points both with non-physicalist versions of behaviorism and with neo-vitalism. Moreover, the importance of his Kantian background suggests relativizing the alleged break between his first publications on invertebrates and his later theoretical work focusing on the notion of Umwelt and on a "semiotic" reinterpretation of biology. However it appears that this semiotics, and the associated concepts of sign (Zeichen), signification (Bedeutung), characteristic (Merkmal), proxy (Repräsentant), are not structured into a coherent program. In a historical context where it was difficult for the still emerging biology to define its specific character between the mechanism epitomized by Loeb and a persistent vitalism, semiotics made it first and foremost possible to model the attributes of life with a minimum explanation.

Keywords - behaviorism, biosemiotics, boundary-concepts, neo-Kantianism, semiotics, Uexküll, Umwelt, vitalism

\section{PRÉSENTATION}

Plongeant une partie de ses racines chez Goethe et dans la Lebensphilosophie, mais aujourd'hui considérée comme l'une des sources de l'éthologie au même titre que celle de K. Lorenz, l'œuvre d'Uexküll (1864-1944) présente quelques traits familiers pour le lecteur moderne. On lui doit notamment la distinction entre l'environnement matériel d'un être vivant (Umgebung) et son Umwelt, son « monde environnant», qui désigne la somme des éléments qui font sens pour lui 
compte tenu de ses propriétés sensori-motrices. Ceci conduit Uexküll à rejeter la croyance en l'existence d'un monde (Welt) unique dans lequel tous les êtres vivants seraient plongés ${ }^{1}$. Alors que, dans l'Umgebung, les vivants sont assimilables à des entités matérielles, chaque umwelt est quant à elle spécifiquement construite et aménagée par un sujet. En soi l'approche écologique du vivant n'était nullement nouvelle au tournant du siècle - elle est constitutive de la notion même de milieu -, mais l'umwelt uexküllienne se distinguait ainsi de la plupart des théories concurrentes, darwinisme compris, et en particulier de la notion de milieu telle qu'on la rencontrait à la même époque dans la tradition française, qui ignorait cette distinction.

Comme la quasi-totalité des travaux descriptifs d'Uexküll sont consacrés aux invertébrés, ils ne font en revanche appel à aucun contenu de conscience, ni à une sémantique. Son œuvre permettait donc de réintroduire le sens, voire la finalité, absents du darwinisme, sous une forme qui semblait compatible avec le béhaviorisme naissant, c'est-à-dire avant son tournant mécaniste ${ }^{2}$. Cette reformulation sémiotique de ce qui s'exprimait ailleurs en termes de stimulus mécanique ou de causalité a ainsi rencontré un écho favorable chez des béhavioristes comme Jennings et Tolman, qui étaient conscients des limites du physicalisme. Rétrospectivement, l'umwelt uexküllienne apparaît plutôt cousine du monde comportemental de $\mathrm{Koffka}^{3}$, ou de l'entourage de comportement merleaupontien. Il n'est pas inutile au demeurant de se rappeler les commentaires rétrospectifs de Merleau-Ponty sur le «manifeste » de Watson :

Lorsqu'il annonce l'étude du comportement, Watson prévoit une nouvelle approche de l'organisme, qui devrait décrire un « courant d'activité » qui attache tout être vivant à l'environnement. On entrevoit une recherche dont le paramètre d'objectivité n'oblige pas à réduire le comportement à des structures physiologiques simples. Il s'agit de circonscrire un entourage de comportement.

Cet entourage doit être compris comme un champ, un milieu de rapports de force et de tensions d'où la conduite des organismes se dégage.

1 Voir Uexküll (1909). Umwelt est le mot le plus fréquemment utilisé par l'auteur pour l'opposer à l'environnement matériel, mais le vocabulaire d'Uexküll a fluctué. La notion s'est quant à elle rapidement stabilisée et la terminologie est restée en retard sur la théorie. Dans ce qui suit, umwelt (sans majuscule) est traité comme un terme et non comme un mot allemand.

2 Une précision : les béhaviorismes (au pluriel !) plongeaient leurs racines dans la tradition européenne et n'ont jamais formé un bloc homogène. L'étude du comportement (Verhaltensforschung) se voulait systémique, en considérant, au rebours d'une conception solipsiste du vivant, que celui-ci n'est pas séparable de son environnement, et elle n'était pas réductionniste, car elle reposait au contraire sur une distinction méthodologique entre un comportement et une relation mécanique de type stimulus-réponse. L'introduction de la notion de behavior avait donc initialement pour but de mettre en évidence les spécificités du vivant, tout en évitant les écueils vitalistes ou anthropomorphistes. C'était là une composante du «manifeste » de Watson (1913). En résumé, que la notion de behavior se soit lestée de telle ou telle idéologie de la science, c'est une chose, qui ne change rien au fait que, comme la plupart des appellations analogues, béhaviorisme ne désigne pas d'office une doctrine, mais plutôt un ensemble de travaux partageant des traits communs, voire regroupés par ressemblances de famille. La distinction entre Umwelt et Umgebung est sans nul doute une spécificité d'Uexküll, mais elle n'est nullement incompatible avec ladite ressemblance. Sur le premier béhaviorisme : Samain 2018a, $2018 \mathrm{~b}$.

3 La distinction qu'effectue Koffka entre behavioral et geographical environment est, sinon semblable, du moins parente de ce qu'on trouve chez Uexküll. Voir en particulier Koffka (1935). 
L'étude des forces en action dans le champ de chaque espèce réfute la thèse selon laquelle les réactions animales sont des réponses à n'importe quel stimulus ; en réalité, la conduite animale est une réponse à des situations particulières; les êtres vivants sélectionnent quelques éléments du monde géographique afin d'établir des ensembles privilégiés de stimuli par rapport auxquels ils agissent. (Merleau-Ponty 2001 : 433-434, souligné dans le texte)

Les lignes d'Uexküll le plus souvent citées concernent le comportement de la tique lors du passage d'un animal à sang chaud. Un comportement qu'Uexküll refuse d'interpréter en termes mécanistes, au motif que, parmi tous les facteurs matériels qui environnent l'animal, seuls deviennent effectivement des stimuli ceux qui sont en quelque sorte traductibles dans son système perceptif :

Ce n'est pas l'excitation chimique de l'acide butyrique qui est en question, pas plus que l'excitation mécanique (provoquée par les poils) ni l'excitation thermique de la peau, mais simplement le fait que parmi les centaines d'effets qui proviennent du corps du mammifère trois seulement deviennent pour la tique des porteurs de caractères perceptifs [...]. Nous n'avons pas affaire à un échange de forces entre deux objets, mais aux relations entre un sujet vivant et son objet, et celles-ci se jouent sur un tout autre plan, c'est-à-dire entre le signe perceptif du sujet et l'excitation provenant de l'objet. (Uexküll 1965: 23; traduction française : Uexküll \& Kriszat 1956 [1934] : 28)

Dans le cadre systémique ainsi posé, Uexküll a simultanément introduit des notions devenues emblématiques de la cybernétique, telle celle de cercle fonctionnel (Funktionskreis), c'est-à-dire la boucle de rétroaction qu'on retrouve un peu plus tard dans les travaux de Wiener et Bertalanffy ${ }^{4}$. En voici deux illustrations simples, qui permettent au passage de comprendre ce qu'Uexküll entend ci-dessus par « caractères perceptifs » (Merkmale) :
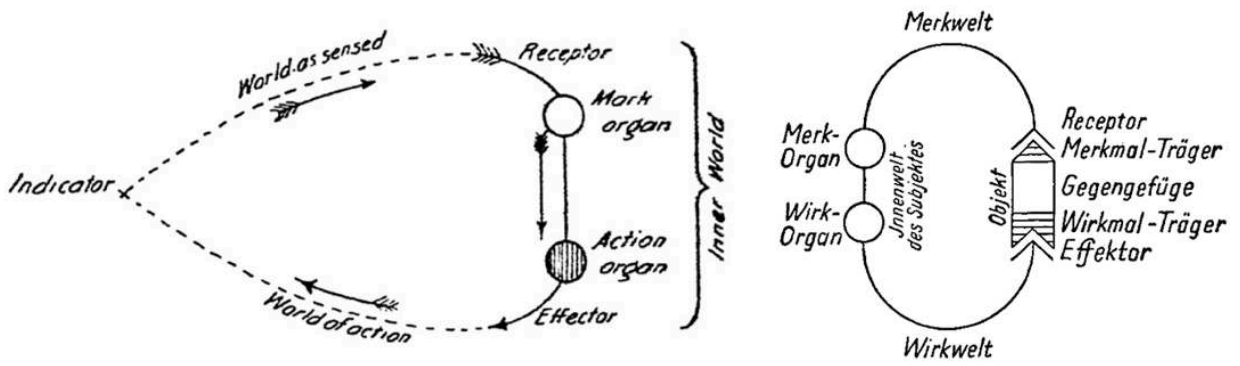

Le schéma de gauche est issu de la première édition de Theoretische Biologie (désormais $\mathrm{TB})^{5}$, son ouvrage épistémologiquement le plus abouti (Uexküll $1920)^{6}$, présenté ici dans sa traduction anglaise $(1926: 155)$ pour en faciliter la

4 Bertalanffy (2006 [1969]) se réfère du reste à de nombreuses reprises aux textes théoriques d'Uexküll.

5 Les références indiquées plus bas sont celles de la seconde édition.

6 Surtout dans sa version remaniée de 1928, ce texte présente de manière détaillée l'essentiel de l'appareil théorique d'Uexküll, alors que les autres travaux sont, soit plus descriptifs, soit plus généralistes et sans doute destinés à un public large. Ajoutons que le titre n'était pas anodin compte tenu des nombreux travaux au titre similaire publiés dans les premières décennies du siècle : outre Uexküll, Reinke 1901, Ehrenberg 
lecture aux non-germanistes. Il a été repris (figure de droite) sous une forme légèrement développée dans la réédition remaniée (non traduite) du même livre (Uexküll 1973 [1928] : 158), et dans plusieurs publications d'orientation théorique ou programmatique (1956 [1934] : $27 ; 1935: 257-272$ ). Contrairement au précédent, le nouveau schéma distingue l'objet comme porteur de caractères perceptifs et l'objet comme porteur de caractères d'effection. Sans entrer dans le détail pour le moment, notons que cette modification montre clairement que, pour Uexküll ${ }^{7}$, cet « objet » n'est pas, ou pas seulement, une chose matérielle (Ding) présente dans l'environnement physique, mais un objet de l'umwelt tel qu'il est perçu ou agi par un sujet donné. Ajoutons que ces schémas ne sont pas chez lui de simples illustrations didactiques, car ils correspondent à des modèles fonctionnels généraux. En particulier ils ne servent pas uniquement à figurer, comme ici, la réponse d'un organisme à un stimulus extérieur, mais tout aussi bien, par exemple, à modéliser le métabolisme intracellulaire.

Quoi qu'il en soit, la thèse constitutive, illustrée par ces schémas, est donc que le vivant sélectionne et transforme en excitations spécifiques les stimuli matériels. Quelles conclusions Uexküll estime-t-il devoir en tirer? Puisque ces stimuli ne sont pas interprétables en termes purement causaux, il en ressort en bref, du moins selon la lecture qu'en font les commentateurs qui se réclament aujourd'hui de la « biosémiotique », qu'il faut les interpréter comme des signes (Zeichen). Or cette inférence peut faire difficulté, car ce qu'Uexküll qualifie de signe ne va pas de soi : si la distinction entre signal et cause matérielle, et celle, corrélative, entre chose et objet (de l'umwelt) paraîtra intuitivement claire au lecteur non prévenu, il est plus difficile de comprendre la nécessité de cette notion de signe, sauf à lui donner une acception très extensive et affaiblie à proportion. La simple sélection des traits pertinents assurant la reconnaissance d'un stimulus implique-t-elle d'office l'emploi d'un métalangage sémiotique ? Et dans l'affirmative, faut-il recourir à la notion générique de signe plutôt qu'à celle, plus technique, de signal ? Car si signe il y a, il semble justement se réduire à un signal et à des affordances. De même, si la notion de « représentant » chez Uexküll a été parfois rapprochée du concept peircien, elle a été critiquée avec l'argument similaire qu'il ne s'agit pas stricto sensu ici de représentation, mais de transformation ${ }^{8}$. Nous reviendrons in fine sur ce point.

1923, Bertalanffy 1932-1942, Rothschuh 1936, pour ne citer que quelques noms, sans oublier les monographies publiées de 1919 à 1931 dans les Abhandlungen zur theoretischen Biologie sous la direction de Julius Schaxel. Telle que l'avait définie Reinke, la «biologie théorique » ambitionnait de parvenir à une caractérisation générale du vivant par un retour méthodologique sur les connaissances biologiques déjà acquises.

7 Ici encore la terminologie utilisée importe moins que le principe lui-même. Dans la version remaniée de sa Theoretische Biologie, Uexküll distingue de manière plus systématique et argumentée entre Ding, Objekt et Gegenstand, mais nous pouvons ici négliger cet aspect.

$8 \mathrm{La}$ Vorstellung, la représentation mentale, étant ici exclue d'office, la question biologique n'en porte pas moins sur la nature du «message » véhiculé par les nerfs reliant l'appareil sensoriel au système central : l'excitation (Erregung) ainsi produite et transmise est-elle une sorte de substitut, de « représentant» (Vertreter, Repräsentant), de l'irritation (Reiz) sensorielle périphérique, ou suffit-il de parler de transformation de cette irritation? De la réponse fournie dépend l'introduction ou non d'un métalangage sémiotique - et réciproquement... Je reviendrai plus bas sur la notion de représentant. 
Pour comprendre le rôle chez Uexküll de la sémiotique ou de quelque manière qu'on l'appelle, il est utile d'en esquisser rapidement le contexte d'activité ${ }^{9}$.

\section{UEXKÜLL, LE VITALISME, ET LA PLANMÄSSIGKEIT}

L'œuvre d'Uexküll a été revendiquée par des courants de pensée antidarwiniens et volontiers néovitalistes et elle est aujourd'hui le plus souvent inscrite dans la perspective biosémiotique. Ces lectures ne sont pas dénuées de fondements et l'évolution d'Uexküll vers une forme de vitalisme est indéniable. Au cours de sa formation, il avait été cependant en contact avec des représentants d'écoles de pensée concurrentes, et ses travaux ont suscité l'intérêt de chercheurs divers. C'est ainsi par exemple qu'un texte de jeunesse, cosigné avec Beer et Bethe (Beer, Bethe \& Uexküll 1899), qui était une sorte de manifeste méthodologique pour une terminologie débarrassée de tout anthropomorphisme, lui aura valu une certaine notoriété auprès des béhavioristes américains. Son hostilité aux approches « mécanistes » du vivant est néanmoins perceptible dès les premiers travaux, consacrés aux oursins ${ }^{10}$. À défaut d'une analyse plus exhaustive, un regard aux références présentes dans TB permet d'esquisser un cadre général, qui est resté globalement stable. Quelques noms, parmi ceux les plus souvent cités ${ }^{11}$, suffiront à confirmer ce que suggérait déjà à sa manière le manifeste de 1899 , à savoir la recherche d'une voie qui éviterait les explications causalistes, celle de Darwin compris, tout en tentant peu ou prou d'échapper au vitalisme classique.

Parmi les contemporains directs d'Uexküll, il faut d'abord mentionner Hans Adolf Eduard Driesch (1867-1941), qui fut son collègue à la station zoologique de Naples. Driesch est connu pour avoir tenté de tester empiriquement la thèse weismannienne du plasma germinatif ${ }^{12}$, et notamment pour une expérience sur les blastomères ${ }^{13} \mathrm{~d}$ 'oursin, dont il était parvenu à scinder les noyaux. Il est apparu que les cellules ainsi divisées se développent normalement, et ne donnent pas naissance à des moitiés d'oursin mais à des individus complets. L'expérience réfutait donc l'hypothèse d'une homologie physique ou géométrique entre la structure de la cellule et celle de l'individu. Driesch en avait conclu que la morphogenèse fait appel à des facteurs non spatiaux, "supramécaniques », et n'avait pas hésité à qualifier d' ' entéléchie » ce facteur organisationnel propre au vivant, qui intervient sur le développement matériel de la cellule sans être matériel lui-même. Il l'opposait aux «mécanismes » que sont les systèmes, tels les

9 Pour la notion de contexte d'activité : Samain 2020.

10 Voir par exemple Uexküll $1896: 9$.

11 Il faudrait en principe en ajouter plusieurs autres dont, au moins, Goethe et Weismann. Mais il ne s'agit ici que de contextualiser rapidement la position d'Uexküll.

12 La différenciation cellulaire au cours de l'ontogenèse conduit Weismann (1892) à postuler que chaque partie obtenue par division contient une part spécifique du matériel héréditaire. Les travaux de Driesch s'appuient sur ceux de Weismann sans les reprendre exactement. Il serait ridicule de chercher dans le plasma germinatif et ces débats d'époque une préfiguration de la découverte de l'ADN, mais force est de constater qu'ils tournent autour d'une question alors insoluble : de quelle nature est l'information transmise par la cellule s'il apparaît qu'elle n'est pas d'ordre purement physique ?

13 C'est-à-dire lors de la toute première division cellulaire (Driesch 1892). 
artefacts, qui fonctionnent sur un mode causal, entièrement déterminé par leurs conditions initiales (Driesch 1919). Ce qui a intéressé Uexküll dans l'expérience de Driesch, qu'il considère comme cruciale, est qu'elle démontrait à ses yeux que le germen n'est pas d'ordre spatial. « Nous savons déjà, écrit-il (TB : 155) en paraphrasant quasiment Driesch, que le protoplasme a pour fonction d'exercer un rôle qui correspond à un plan [planmäßig $\left.{ }^{14}\right]$, mais qui est supramécanique [übermaschinelle].» En bref, nous dirions aujourd'hui que le protoplasme transmet une « information » qu'à défaut de pouvoir définir, Uexküll déterminait négativement.

Il est instructif de comparer au passage les thèses de Driesch et celles de la Gestalt, qui lui sont contemporaines et dont il partage la perspective holistique ${ }^{15}$. La geordnete Ganzheit, la totalité organisée, se distingue d'une gestalt selon Driesch sur deux points. Il y voit d'une part une spécificité du vivant et, d'autre part, il ne la considère pas comme une réalité matérielle, c'est-à-dire spatiale, contrairement justement à l'école dominante de la Gestalt. On retrouve les mêmes arguments dans TB, qui oppose, tout comme Driesch, la réalisation mécanique effectuée d'après un patron ou un modèle (Schablone) et ce qui suit une règle (Regel), un mot qu'il faut ici comprendre plutôt au sens de "programme », en relation à la Planmäßigkeit. "Est-ce que l'achèvement d'un objet se réalise de manière obligatoire en suivant un patron matériel, ou se produit-elle en vertu d'un plan [planmäßig] suivant une règle déterminée?» (TB : 234). Pour Uexküll comme pour Driesch, la conformité à un plan est une spécificité du vivant, mais Planmäßigkeit n'implique pas intelligent design, et le champ disciplinaire qu'il se propose de délimiter, à savoir la biologie, doit se tenir à distance de la psychologie comme de la physique. L'umwelt de l'animal n'est pas la nôtre, écrit-il (TB : 155). C'est donc notre aperception qui perçoit la Planmäßigkeit ${ }^{16}$, et nous ne pouvons rien en extrapoler quant à l'esprit de l'animal. Uexküll emprunte pour la circonstance à Driesch la notion de «psychoïde », par lequel ce dernier désigne le facteur «supramécanique » dont l'observateur infère, par les effets observés, qu'il est analogue à son propre système perceptif. Le psychoïde est donc le nom de l'agent élémentaire qui se révèle dans une action (ibid.). Tout comme la masse aperceptive de la psychologie empirique, le psychoïde a une épaisseur temporelle, ce qui le rend susceptible d'apprentissage, contrairement à une machine (TB :

14 Quand il s'agit de l'auto-organisation du vivant, le vocabulaire d'Uexküll est (volontairement ?) souvent ambivalent. Planmäßig ne doit pas ici se comprendre comme signifiant en français «planifié », ce qui suggèrerait que ce «plan » émane d'une source extérieure. Nous voyons à quel point Driesch, Uexküll, et de manière générale le néo-vitalisme, marchaient sur une corde raide. Cette corde raide, et fil directeur, est la Planmäßigkeit, la « conformité à un plan », une expression récurrente dans l'œuvre. La découverte de l'ADN, puis, plus tard, les réseaux de neurones artificiels et le deep learning ont naturellement changé la donne.

15 Ce que fait du reste Uexküll lui-même (TB : 293) en prenant parti pour Driesch. La plupart des gestaltistes considèrent les gestalts (les formes) comme des propriétés sui generis des objets eux-mêmes. Mais ce point de vue était rejeté par des courants minoritaires, inspirés de Meinong et de Kant.

16 Uexküll évoque par exemple à plusieurs reprises «l'accord» (dans un sens quasi musical) entre la mouche et la toile de l'araignée. Pour l'araignée, qui a construit sa toile guidée par son instinct et sans avoir jamais vu une mouche, celle-ci n'est qu'une tâche sombre associée à des vibrations tactiles. La conjonction de ces deux caractéristiques (Merkmale) signifie «proie ». 
180). Outre sa prudence terminologique, pareil propos n'avait rien qui pût scandaliser un théoricien de l'analyse comportementale, Verhaltensforschung, Behaviorism, pour autant que ce béhaviorisme n'optait pas pour le dogmatisme physicaliste, ce à quoi rien ni personne ne l'obligeait. - C'est l'observateur qui perçoit la Planmäßigkeit ${ }^{17}$. - Derrière cette Verhaltensforschung telle que pratiquée par le biologiste pointait un fond de kantisme, également suggéré par les schémas précédents, lesquels disent clairement que l'objet perceptif (Merkding) situé dans l'espace perceptif (Merkraum) spécifique à une umwelt n'est pas la chose matérielle. «L'objet perceptif est un produit du psychoïde, tout autant pour sa forme que pour son contenu », précise Uexküll (TB : 181). La théorie du psychoïde, le fait que les analyses d'Uexküll ont majoritairement porté sur des animaux très simples, nous assurent qu'il ne s'agit pas davantage d'une représentation mentale.

Avant d'en venir à Kant, deux mots sur une autre source d'Uexküll, antérieure à Driesch, en l'occurrence Karl Ernst von Baer (1792-1876), qui fut sans doute l'un des derniers représentants du vitalisme classique ${ }^{18}$. Baer est en effet, avec Driesch, l'une des références les plus souvent citées par Uexküll, et la proximité entre les deux auteurs transparaît à plusieurs niveaux. D'abord, globalement, dans leur rejet du darwinisme et de toute interprétation mécaniste, purement causale, du monde animal, mais aussi à un niveau de généralité moindre, celui des idéologies scientifiques et des analogies heuristiques ou explicatives. On trouve ainsi chez eux un même recours « explicatif » récurrent à la métaphore musicale pour figurer les processus vitaux et l'autopoièse du vivant. Exemple chez Baer :

Dans les organismes, les parties constituantes sont construites en fonction du type et du rythme des processus vitaux concernés et des effets produits, de sorte qu'ils ne pourraient servir à un autre processus vital. C'est la raison pour laquelle, en les comparant à des pensées et des thèmes musicaux, je crois pouvoir les qualifier de pensées créatrices qui édifient elles-mêmes leurs propres membres. Ce que nous appelons en musique harmonie et mélodie, c'est ici le type (la coprésence des parties) et le rythme (la consécution des formes). (Baer $1864: 280-281$ )

Dans TB, Uexküll se réfère à Baer pour comparer la régularité de la mélodie et celle de la formation de l'embryon (TB : 221), il parle aussi de Symphonielehre

17 Voir ce commentaire de Jacques Fontanille : « Du point de vue de l'observateur humain, les perceptions et les actions sont articulées en séquences canoniques porteuses du sens global de la pratique animale, chacune étant précisément située en une phase déterminée. Mais, de son point de vue, la tique n’a affaire qu'à un schème d'interaction, c'est-à-dire à une pression somatique et sensible qui l'incite à enchaîner les interactions entre perceptions et actions. [...] Une fois que la séquence régulatrice est reconnue par l'analyste, elle acquiert une stabilité suffisante pour se prêter à toutes sortes de manipulations : saisie à partir de la fin, elle se donne comme un "destin" » (Fontanille 2019).

18 Tout à la fois géologue, géographe, biologiste, etc., Baer fut l'un des scientifiques majeurs de son siècle, à qui on doit, entre autres, d'avoir montré que le développement embryonnaire des animaux s'effectue par spécification progressive, ce qui réfutait la thèse de la récapitulation. Défenseur, comme Uexküll, d'une interprétation téléologique de l'évolution, il s'est opposé à la théorie de la sélection naturelle. Il était né à Dorpat et il est revenu dans sa ville natale en 1867, après avoir effectué l'essentiel de sa carrière à Königsberg (la ville de Kant !). Pour des raisons biographiques évidentes, Uexküll ne peut y avoir suivi son enseignement, mais la géographie a sans doute contribué à la filiation théorique. Sur la filiation Uexküll-Baer : Kull 2001. Sur Kant et la téléologie, voir ci-dessous. 
des Schauens à propos de la vision (TB : 46), de Baummelodie (TB : 184), de Bewegungsmelodie (TB : 187), etc. ${ }^{19}$ À un degré similaire de généralité, il a par ailleurs emprunté à Baer le couple Ziel/Zweck ${ }^{20}$, qui éclaire au passage sa notion de Planmäßigkeit. Le Zweck, écrit-il (TB : 319), est une représentation posée dans le futur, un objectif, dont rien ne garantit au demeurant la complète réalisation. Et de résumer la position de Baer, qui considère qu'il n'y a pas de Zweckmäßigkeit (entendons de finalité consciente) dans la nature, et qui opte pour Ziel, qu'il juge plus neutre. Baer, et Uexküll à sa suite (ibid.), évoquent ainsi la relation de « tension vers la cible » (Zielstrebigkeit) qui relie le couple formé par la cartouche et sa cible. La « tension vers la cible » désigne un couple fonctionnel, défini sans référence au tireur. Ce détail n'est pas entièrement anodin, confirmant le choix d'un engagement ontologique réduit.

Un troisième niveau de généralité concerne les propositions locales, c'est-àdire des énoncés techniques, directement corrélés à une réalité empirique donnée. Il en est un exemple fort intéressant, car il concerne un composant théorique important de la théorie biologique d'Uexküll, à savoir la façon dont le système perceptif de chaque vivant discrétise de manière spécifique le continu physique. Uexküll emprunte ici à Baer sa notion de moment, que ce dernier définit comme le plus petit intervalle de temps perceptible pour un vivant donné. Cette définition du moment implique que la durée phénoménologique est constituée d'espaces de temps discontinus non compressibles, et que le «moment » est donc une qualité temporelle spécifique ${ }^{21}$. «L'aperception, écrit de son côté Uexküll (TB : 70), est un processus vital qui se déroule en phases, au cours duquel chaque phase s'exprime par un signe sensible. Ce signe est un moment. » Retenons que le moment sémiotique correspond à une transposition (une traduction si on y tient) du continu physique en discontinu phénoménologique. Il ne s'agit pas simplement de l'image éculée d'un continuum temporel, ou d'un continuum perceptif quelconque, segmenté à la manière éléate, mais de la thèse (non triviale) qu'une sémiotisation achevée correspond à la transposition des impressions sensorielles physiques (les excitations) en données qualitatives.

$19 \mathrm{Ce}$ ne sont que quelques exemples illustratifs. On retrouve le même motif dans les autres travaux théoriques, par exemple dans Uexküll (1909 : 28-29, 178) et (1956 [1934],passim). La distinction mélodie/ harmonie mériterait au demeurant quelques commentaires, impossibles ici.

20 Ces deux mots signifient «but», mais Baer a exploité une différenciation tendancielle dans l'usage ordinaire, car les contextes de Zweck sont plus fréquemment associés à l'idée d'intention, tandis que ceux de Ziel soulignent plutôt le résultat ou le point d'arrivée. - Ziel peut également désigner une " cible », comme dans l'exemple qui suit. Plus tard, Uexküll (1956 [1934] : 60) rejettera en revanche Ziel, mais parce qu'il y associera cette fois l'idée d'intention. Ailleurs (1905c : 94-95), il parle au contraire de Naturzwecke (i. e. appartenant au domaine de la biologie) vs Naturprodukte (i. e. domaine de la physiologie). Comme précédemment le vocabulaire importe donc assez peu et sert surtout de support. Dans cette suite de parallaxes, certaines désignations sont néanmoins plus stables, moins décentrées. C'est le cas de Planmäßigkeit.

21 De ce point de vue les thèses de Baer sont un jalon dans le développement de l'approche phénoménologique de la durée. Sur l'importance de Baer : Pöppel 2004, Kull 2015. Sur le temps phénoménologique et le « specious present », voir Arstila \& Loyd 2014. 
Venons-en à Kant. Sans doute l'umwelt uexküllienne présente-t-elle en soi une coloration kantienne ${ }^{22}$, mais cette affinité ne se limite pas à une similitude qui pourrait passer pour fortuite. Uexküll considère en effet les trois Critiques comme une sorte de boîte à outils méthodologique ${ }^{23}$, et ses références à Kant sont récurrentes (1902, 1905c, 1907a, 1907b, 1920, 1973 [1928], 1956 [1934], etc.). TB commence même par une longue discussion du programme kantien et en particulier de la deuxième partie de la Critique de la faculté de juger (§ $61 \mathrm{sv}$.), consacrée au jugement téléologique et à l'opposition entre organique et mécanique. Même si, comme nous le verrons dans un instant, Uexküll s'écarte de Kant sur plusieurs points, la conception critique de la notion de finalité développée par le philosophe de Königsberg ne pouvait qu'intéresser le biologiste balte, cherchant à mieux définir la Planmäßigkeit ${ }^{24}$. Uexküll trouve dans la troisième Critique plusieurs des traits censés caractériser le vivant : la corrélation (dans l'acception stricte d'implication réciproque) entre le tout et ses parties, la distinction entre Kausalität (causalité mécanique) et Zweckmäßigkeit (équivalent chez Kant de la Planmäßigkeit d'Uexküll). Dans un passage célèbre (1922 [1790] : 237), Kant distingue ainsi l'arbre et la montre, dont chaque partie est certes cause du mouvement de toutes les autres, mais qui n'est pas la cause effective de leur propre existence, laquelle cause se trouve à l'extérieur de la montre, dans l'horloger. L'arbre par contraste s'engendre lui-même, possède, dit Kant, une bildende Kraft, une capacité formative dont la montre est dépourvue. Ce type d'argument se retrouve chez Uexküll ${ }^{25}$.

Toutefois, si le kantisme fournit une charpente théorique générale, il subit une transposition qui en modifie l'esprit. Dès 1907 (1907b: 658-659), Uexküll esquisse une reformulation biologique, peu ou prou empiriste, des propositions de Kant, qui s'est poursuivie dans l'œuvre ultérieure. TB se veut ainsi un développement du kantisme dans deux directions (TB : 55-70). D'une part, en élargissant les catégories kantiennes au-delà des seuls temps et espace, et d'une manière qui prenne en compte le rôle du corps et des organes des sens ${ }^{26}$. D'autre part, en

22 TB : 46 : «Le secret du monde ne doit pas être cherché derrière les objets, mais derrière les sujets. »

23 Exemple : « Nous disposons depuis plus de cent ans des trois grands travaux critiques de Kant, qui ne sont pas un système philosophique, qui contiennent un examen scientifique [naturwissenschaftlich] des lois qui régissent la vie de l'esprit humain. Il serait de circonstance, en nous appuyant sur cette base, de procéder ici par expérimentation » (Uexküll 1905c : 130). Tout est dit. Le kantisme fournit un principe structurant, il s'agira de le valider empiriquement.

24 Kant distingue la finalité (Zweckmäßigkeit) réelle, dans le cas d'un jardin par exemple, de la finalité formelle, qui caractérise le vivant et ne doit être considéré que comme un outil heuristique - une distinction très voisine de celle établie par Uexküll entre la Planmäßigkeit du vivant et celle de la machine. Une remarque incidente : Goethe possédait un exemplaire de la Kritik der Urteilskraft dont il avait souligné les passages consacrés au jugement téléologique. Kant est ainsi l'un des fils qui relient Goethe et Uexküll, à côté d'autres plus visibles, à commencer par une phénoménologie de la nature. Sur un empan historique plus large, tout cela esquisse un frayage qui va peut-être de Kant jusqu'à, aujourd'hui, quelqu'un comme S. J. Gould.

25 Voir par exemple Uexküll (1905c : 66-67).

26 Uexküll (TB : 109) suggère que la limitation aux catégories Raum et Zeit est conjoncturelle, et tiendrait au fait que les autres catégories ne sont pas représentées dans le lexique ordinaire, dépourvu de mot pour les exprimer. L'espace kantien se complexifie par ailleurs chez Uexküll en \{moi/étendue/espace\}. 
étendant aux autres «sujets », en l'occurrence les animaux, l'analyse de la relation du sujet à ses objets.

TB se propose donc de déterminer l'ensemble des facteurs qui, réunis, construisent le monde subjectif d'un vivant déterminé. Ce monde (TB : 95 sv.) consiste en lieux (Orten) de nombre fini, en mouvements (Bewegungen) d'ampleur limitée, en moments dont la série possède début et fin, en qualités de contenu (Inhaltsqualitäten) dont le nombre est de même déterminé et dont les lois sont simultanément des lois de nature (Naturgesetze). Comprenons que, pour Uexküll, l'espace phénoménologique est multidimensionnel, que ces dimensions sont déterminées par les spécificités physiologiques de chaque vivant, et qu'elles ne sont pas indéfiniment divisibles ${ }^{27}$. En bref, le kantisme est bien chez Uexküll un principe structurant, fournissant la charpente théorique, qui rencontre ${ }^{28}$ les observations biologiques dans lesquelles il est transposé.

Le sujet transcendantal se mue donc en sujet biologique, c'est-à-dire en agent réel, qui construit activement son umwelt. L'œil immobile ne fournit pas un espace visuel, écrit par ailleurs Uexküll (TB : $27 \mathrm{sv}$.), mais uniquement une surface visuelle, de même que le corps immobile ne fournit qu'une surface tactile. L'espace est construit par nos mouvements et non par nos perceptions. Pas d'étape de perception (Merkschritt) qui ne s'accompagne d'une étape d'effection (Wirkschritt) (ibid.). Cette transposition biologique s'accompagne d'une reformulation un peu ambiguë d'un couple kantien. Uexküll considère, tout comme Kant, que les notions de causalité et de finalité sont complémentaires. Transposées dans le couple Umgebung/Umwelt, elles correspondent à la différence entre physiologie et biologie. Toutefois, dans la philosophie transcendantale, cette complémentarité se fonde sur une différence de principe : la causalité relève de l'activité constitutive de l'intellect (Verstand), alors que la Zweckmäßigkeit ${ }^{29}$ est le fruit de l'usage régulateur de la raison (Vernunft) (Kant 1922 [1790] : 293). Le recours à cette dernière est donc d'abord heuristique. Uexküll en revanche, qui se réfère à nouveau à Driesch (TB : 293), reproche à Kant de n'avoir vu dans le plan qu'une simple règle humaine ajoutée mentalement ${ }^{30}$, alors que Driesch voit dans

27 Sur la divisibilité de l'espace phénoménologique, voir ci-dessus note 21. Par un chemin différent, le propos d'Uexküll rejoignait ici l'une des intuitions les plus stimulantes de la Gestalt.

28 Sur la notion de « rencontre » entre principes structurants et propositions locales : Samain 2020.

29 Ou Planmäßigkeit. Quoique moins fréquent que Zweckmäßigkeit, ce terme apparaît aussi dans la Kritik der Urteilskraft, mais je dois ici négliger cette variation. On peut du moins penser que, chez Uexküll, la progressive prévalence de Plan sur Zweck n'est pas fortuite (voir ci-dessus, note 20).

30 Dans ses Studien über das Tonus, Uexküll adoptait en revanche une position plus strictement kantienne : «Observer les choses en y voyant des buts de la nature relève de l'usage régulateur de la raison. [...] Les deux modes d'observation sont des expressions pareillement indispensables de l'esprit humain », écrivaitil, critiquant « l'erreur des vitalistes », qui « ont voulu faire un usage constitutif d'une fonction régulatrice » (Uexküll 1905b : 376). Ceci peut se comprendre de deux manières, qui ne sont pas incompatibles entre elles : soit comme le signe d'un glissement vers le vitalisme à partir de 1920, soit comme l'indice que la Planmäßigkeit a pris peu à peu chez lui la valeur d'un concept frontière. Nous constatons au passage l'importance des concepts frontières dans la dynamique des sciences. On est facilement tenté de tracer une cloison étanche entre, par exemple, un principe constitutif et un principe régulateur, entre neurologie et psychologie, entre réalisme et conventionnalisme, etc. Dans la pratique, les choses ne se passent pas exactement ainsi. Non qu'il y ait une transition progressive d'un domaine à un autre, car ces domaines sont 
la Planmäßigkeit une propriété constitutive. Nous retrouvons notre corde raide et fil directeur. - Certes, la Planmäßigkeit du vivant n'est pas une propriété physique de la substance, ni une simple structure. Pour Uexküll elle n'en est pas moins dans le réel, et non pas seulement dans l'esprit du sujet.

\section{UEXKÜLL ET LES BÉHAVIORISTES}

Les travaux d'Uexküll sur la physiologie du réflexe étaient connus des contemporains et en phase avec leur époque. Dans son article consacré à la réception de l'œuvre d'Uexküll, Mildenberger (2006) signale même des anecdotes inattendues pour le lecteur d'aujourd'hui - le fait par exemple que sa candidature (infructueuse) au poste de directeur de l'Institut Kaiser Wilhelm en 1918 avait reçu le soutien de Loeb, quelqu'un qu'il eût été pour le moins difficile d'enrôler dans le néokantisme ${ }^{31}$. Mildenberger y voit le résultat d'un malentendu : selon lui, les travaux vitalistes d'Uexküll ont été tout simplement ignorés, et son effacement de l'horizon académique américain dans les années soixante tient au recul du béhaviorisme lui-même, et non au fait que ses représentants auraient remarqué son évolution. Cette analyse est sans doute juste, mais il ne faudrait pas en conclure à une discontinuité radicale chez Uexküll entre des travaux expérimentaux et une œuvre théorique ultérieure influencée par Kant et le néovitalisme, ni du reste entre celle-ci et les positions béhavioristes.

S'agissant du premier point, la tentative de transposition biologique du kantisme vient d'être mentionnée. Mais les références à Kant dans les travaux descriptifs de jeunesse sont tout aussi explicites que dans l'œuvre théorique ultérieure. Voici un autre fil directeur. Dans un essai consacré au tonus musculaire chez les invertébrés, publié en plusieurs articles successifs (Uexküll, 1903, 1905a, 1905b, 1907a, 1908, 1912), Uexküll propose de représenter l'influx nerveux par un «fluide», tout en prenant quelques précautions : la «recherche directe» (c'est-à-dire la physiologie), explique-t-il, se donne comme objectif d'établir la nature de l'excitation nerveuse, mais la « recherche indirecte » (soit la biologie), qu'il promeut, et qui

veut simplement réunir un ensemble de matériaux comparables à partir des expérimentations sur les relations d'excitation chez des animaux divers, intègrera

réellement disjoints. Mais parce qu'on rencontre, au voisinage des limites, des notions bifrons, justiciables de deux interprétations normalement incompatibles. C'est le cas chez Uexküll de la Planmäßigkeit. Correctement interprétée, la notion de principe régulateur est du reste elle-même un concept frontière, puisqu'elle permet justement de suspendre cette alternative entre réalisme et conventionnalisme. Ou, pour dire les choses autrement, dans les théories scientifiques, les concepts frontières font figure de migrants en attente sans fin de régularisation, alors qu'ils jouent un rôle heuristique (dynamique) important, pour deux raisons au moins : en faisant office de point de contact entre champs disciplinaires d'une part, en permettant de continuer à raisonner tout en suspendant le jugement de vérité d'autre part.

31 Loeb (1859-1924), connu notamment pour avoir appliqué la notion de tropisme aux animaux supérieurs, fut en son temps un représentant typique du " mécanisme»; ou, dans le langage d'aujourd'hui, du réductionnisme. Il partage avec Uexküll une conception systémique du vivant, en concevant l'organisme comme une unité intégrée au sein d'un environnement. Mais il s'agit chez lui d'un environnement purement matériel - Umgebung chez Uexküll, qui le qualifiera plus tard de « physicien viscéral, qui ne reconnaissait que des interactions entre objets, et ne voulait rien savoir d'une influence des sujets sur le fonctionnement de la nature » (1956 [1934] : 123, ma traduction). 
l'expression de toutes les opinions sur la nature de l'excitation. Elle ne pourra en revanche se passer d'un schéma pourvu d'une validité générale, de manière à regrouper dans un cadre unitaire ces expériences disparates. Le schéma de la recherche indirecte n'est pas une théorie, mais juste un langage symbolique [Zeichensprache], qui lui permet d'exprimer aisément sous forme intuitive [anschaulich] les nouvelles expériences. (Uexküll 1905a : 31)

Tout cela appelle plusieurs observations. Observons d'abord que cette distinction entre deux recherches correspond selon Uexküll à celle entre usage constitutif de l'intellect (qui identifie des causes) et usage régulateur de la raison (soit le recours critique à la finalité). Remarquons ensuite que le propos affiche un œcuménisme épistémologique, associé à la recherche d'un langage intégrant, applicable aux diverses espèces et situations - une volonté qu'on retrouve dans le béhaviorisme. Plus fondamentalement enfin, nous retrouvons une corde raide déjà rapidement évoquée plus haut, qui concerne le statut des schémas et des désignations. En effet, interpréter les réflexes en termes de tonus n'était pas anodin et il ressort de cette série d'articles qu'Uexküll désigne par cette notion une réalité frontière, matérielle sans doute, mais sans qu'il lui soit attribué de corrélation physiologique déterminée ${ }^{32}$. Uexküll l'applique du reste aux nerfs tout comme aux muscles.

À proprement parler, le concept de tonus n'autorise aucune inférence concernant sa nature. [...] Il ne permet aucune analyse physique, et il n'est pas davantage accessible à une formulation mathématique. C'est un pur concept biologique, c'est-à-dire un simple schéma, d'après lequel il est possible de regrouper synthétiquement [übersichtlich] les faits découverts expérimentalement. (Uexküll 1903: 287)

Le même constat s'applique au " fluide » censé représenter l'influx nerveux, qui est lui aussi une désignation fonctionnelle, ne préjugeant pas de la nature physique de l'échange opéré. Et il en va de même de la représentation du système nerveux dans son ensemble. Extrait d'un texte de la même période :

Faisons expressément référence à une particularité car elle prête facilement à malentendu. Ce que nous désignons biologiquement comme réseau central n'a souvent rien à voir avec l'entité définie anatomiquement comme système nerveux central. Car, du point de vue biologique, le réseau central est l'organe de liaison des représentants [der verbindende Organ der Repräsentanten], il peut très bien s'étendre jusque dans ce qu'on appelle les nerfs périphériques quand les représentants sont restés situés près des muscles. (Uexküll 1905c : 55)

Ces propos faisaient au passage écho à la distinction entre homologie évolutive et isomorphie fonctionnelle, une problématique d'époque ${ }^{33}$. S'y sont greffées des

32 Voir ci-dessus, note 30, sur la fonction des concepts frontières.

33 Pour mémoire, en théorie de l'évolution, on désigne comme isomorphie, ou « analogie », une similitude fonctionnelle (l'aile des oiseaux et des chauves-souris par exemple) et on appelle « homologie » un trait évolutif partagé par des espèces génétiquement apparentées (tel le nombre de vertèbres cervicales, qui est commun à tous les mammifères, homme, baleine ou girafe), quelles qu'en soient les variations phénotypiques. La « morphologie » est quant à elle l'étude des faits d'homologie. Ces questions - qui concernent donc, en simplifiant les choses et en les traduisant en langage ordinaire, les rapports entre données observables, fonction et genèse, entre synchronie et diachronie - font l'objet d'importants débats à la fin 
conditions expérimentales particulières. - La présence de similitudes fonctionnelles, nonobstant la diversité des architectures matérielles, invitait à chercher des formulations intégrantes. Plus radicalement encore, la mise en évidence de comportements coordonnés et unitaires chez des animaux dépourvus de coordination nerveuse centrale imposait de repenser profondément l'architecture de ces systèmes ${ }^{34}$. Le résultat est que, dès les travaux de cette période, Uexküll a recours à des figurations qui ressemblent parfois davantage à des schémas électriques qu'à des planches anatomiques. Simultanément, ses formules maintiennent souvent une légère ambiguïté. "Quand par exemple je trace le plan structurel [Bauplan] d'un ver, écrit-il, et que j'utilise en l'occurrence un schéma physique quelconque, je ne songe aucunement à m'engager trop avant ${ }^{35}$ dans une problématique physique» (1905b : 377). Il faut donc ici être prudent. Comme nous le verrons, la caractéristique la plus troublante, et peut-être la plus intéressante, des schémas d'Uexküll est justement qu'ils semblent garder une part de réalisme, et que la relation avec la physiologie s'y trouve plutôt transposée que totalement rompue. Rien d'étonnant que ces schémas aient pu inspirer Bertalanffy, puisqu'en cybernétique aussi, une isomorphie avec le monde physique est implicitement postulée ${ }^{36}$. Quoi qu'il en soit, on observe donc une certaine continuité entre les travaux descriptifs des années 1900 (voir les références à la fin de cet article) et Theoretische Biologie. L'introduction de la notion d'umwelt a cependant modifié la perspective. Alors que le kantisme servait jusqu'alors d'outil méthodologique pour le chercheur, il sert, avec cette notion, à modéliser l'activité cognitive des vivants eux-mêmes.

En ce qui concerne le second aspect, le rapport d'Uexküll au « béhaviorisme », les choses sont également moins tranchées qu'il n'y paraît. Comme cela a été signalé plus haut, il faut en tout état de cause distinguer ce qui a été désigné, de

du XIX ${ }^{\mathrm{e}}$ et au début du XX $\mathrm{X}^{\mathrm{e}}$ siècle, qu'Uexküll évoque dans un sous-chapitre de TB consacré à la «morphologie », « science des signes de genèse » (Wissenschaft der Entstehungszeichen), et parsemé d'allusions transparentes aux thèses de Driesch et de Haeckel (TB : 226-234). Tout cela débordait naturellement les théories de l'évolution : s'agissant de la «morphologie », il est difficile de comprendre l'introduction par Schleicher de ce mot en linguistique si on ignore ce contexte.

34 Uexküll qualifie l'oursin de « république de réflexes » (TB : 283). La métaphore de l'État est utilisée avant Uexküll pour décrire le vivant (Haeckel, Virchow, Verworn), mais son originalité est de l'appliquer à des animaux, et dans une perspective qu'on qualifierait aujourd'hui d'émergentiste.

35 Je souligne. Cette nuance disparaît de la traduction anglaise qu'en a donnée Jennings (1909) dans son commentaire d'Uexküll.

36 Les problèmes posés par la figuration schématique chez Uexküll sont probablement plus complexes que cette présentation succincte peut laisser supposer. Chien (2004) signale le contre-sens commis selon lui par Uexküll lorsqu'il se réfère au schématisme kantien. Nul doute en effet que les schémas d'Uexküll ne sont pas plus des schèmes dans l'acception kantienne du terme qu'ils ne sont des ersatz de planches anatomiques, mais cela n'implique pas qu'ils soient purement fonctionnels. En ce qui concerne leur type d'abstraction et leur degré de réalisme, sans doute mériteraient-ils d'être comparés à d'autres schématismes qui reposent sur des paradoxes voisins. On songera à la Gestalt, ou encore, en géographie, à la chorématique de Brunet par exemple. Dans chacun de ces cas, l'ambition reste de figurer le réel tel qu'en lui-même, sans l'aplatir à l'excès - et sans formalisme. Répondant, chez Uexküll, à une volonté (goethéenne sans doute) de ne pas se résoudre à une représentation dématérialisée du monde, un monde qui, écrit-il, «si objectivement qu'il se comporte, n'est plus guère [alors] qu'un moulage dilué [verdünnt] de l'umwelt » (TB : 338). Nous sommes probablement de nouveau en présence d'un phénomène de frontière, analogue à celui dont la description a été esquissée plus haut. 
manière plus ou moins polémique, par ce mot, et ce qu'impliquaient initialement des mots comme behavior ou Verhalten(s-forschung) ${ }^{37}$. Une thèse initiale corrélée à la notion de behavior était que l'organisme se distingue d'une structure inerte par un ensemble de caractéristiques qu'il est possible de regrouper sous celle d'activité orientée, de directive behavior : maintenance, reproduction, et, de manière plus générale, de réponse organisée (i.e. non mécanique) et homéostatique aux stimuli. Ce qu'Edward Russell (1887-1954) résuma un peu plus tard, dans un bref article du premier numéro de Nature :

[...] behavior is simply one form of the general directive activity of the organism; it is that part of it which is concerned with the relations of the organism to its external world. [...] Whether of plants or animals, $[i t]$ is $[\ldots]$ to be regarded simply as one form of the general directive activity which is characteristic of the living organism. It holds no privileged position; it does not require "mind" as an immaterial entity to explain it. (Russell 1934 : 836-837)

Ces comportements, poursuit Russell (ibid.), sont strictement analogues à ceux que, dans l'expérience immédiate, nous décririons comme psychologiques. Les animaux perçoivent le monde extérieur et y réagissent d'une manière qui leur est propre. Chez eux comme chez nous, la perception peut être considérée comme une fonction de l'organisme, non de «l'esprit».

Simple observation shows us that the actions of animals have for the most part a definite objective; they aim towards some specific "end-state", and cease when this is attained. [...] At the same time, we can infer from the character of the action the kind of valence possessed for the animal by the objects and events in relation to which its action is carried out. We may speak therefore of food-valences, danger-valences, mate-valences, parent-valences, offspring-valences, and so all. (Russell 1938 [1934] : 180)

Ces propos de Russell résument un consensus méthodologique, qui persiste tout au long de la période, sur ce qu'il faut entendre par « comportement » et sur la manière de l'aborder. On le retrouve, valence et homéostase comprises, chez Uexküll, dont l'œuvre présente donc des traits indiscutablement béhavioristes, à commencer par l'absence d'hypothèses sur les états cognitifs, doublée d'une approche inférentielle des stimuli. Puisque les systèmes perceptifs des êtres vivants nous sont inaccessibles, dit en substance Uexküll, c'est aussi le cas de leurs signes de perception (Merkzeichen) et d'effection (Wirkzeichen). Ces derniers ne peuvent être identifiés qu'à partir du comportement observable. Ce phénoménalisme comportementaliste se voulait un métalangage unifié, applicable

37 Je n'ai relevé qu'une seule référence explicite au « béhaviorisme » chez Uexküll lui-même (et non chez Kriszat ou ses commentateurs ultérieurs). Elle se trouve dans la préface de la première édition des Streifzüge..., à quoi l'auteur ajoute en conclusion une exemplification parodique de la notion d'umwelt : « Dans l'umwelt de la nature [telle que la conçoit] le béhavioriste, le corps produit l'esprit, et dans le monde du psychologue, c'est l'esprit qui construit le corps » (1956 [1934] : 101, ma traduction). - Dans un texte assez tardif donc (1934), où l'emploi des guillemets (« Behaviorismus », ibid. : 161) signale clairement que l'auteur désigne par là un mouvement géographiquement et temporellement circonscrit, dont il rejette le physicalisme et l'ignorance corollaire des umwelten. J'ai expliqué rapidement en introduction (voir note 2) pourquoi cet usage polémique des appellations ne suffit pas pour l'historien. Voir aussi par contraste l'emploi, plus descriptif, de behavior dans Uexküll 1913. 
sans distinction à toutes les espèces, qui devenaient ainsi commensurables. Chez Uexküll, c'est là l'une des caractéristiques du métalangage biologique.

La recherche des marques caractéristiques ne peut être couronnée de succès que lorsqu'on a étudié les différentes réponses d'un animal dans sa vie normale, car c'est la différence de la réponse qui nous fournit l'information la plus fiable sur la diversité des caractéristiques. (TB : 189)

En amont du courant béhavioriste proprement dit, mais en partie enrôlé par lui, Herbert Jennings (1868-1947) est quant à lui un strict contemporain d'Uexküll qui, peu de temps avant les expériences de ce dernier à Naples, conduit une série de recherches sur le comportement des unicellulaires. Quoique menées dans une perspective plus classique, les expériences de Jennings montraient en particulier que les réponses aux stimuli varient selon les espèces et peuvent inversement rester identiques chez une même espèce quel que soit le stimulus. Il en ressortait que ces réponses sont donc spécifiques (liées à un organisme particulier), et ne sont pas entièrement réductibles à des mécanismes physico-chimiques. Jennings est par ailleurs parvenu à mettre en évidence une capacité d'apprentissage chez les protozoaires, et en concluait que leur comportement présente des caractéristiques (en termes de perception, de sélection, etc.) qui ne diffèrent pas substantiellement de ce qui est observé chez les animaux supérieurs ${ }^{38}$.

Ces thèses ne divergent guère de celles d'Uexküll, et les deux auteurs, qui travaillent sur des domaines voisins, se citent réciproquement. Jennings publie toutefois en 1909 un article spécifiquement consacré à Uexküll qui se veut une sorte de présentation critique des thèses de son collègue depuis les années quatrevingt-dix. Jennings souligne certains apports originaux de la réflexion d'Uexküll, tels la notion de tonus, le fonctionnement des réflexes coordonnés chez les animaux dépourvus de centre organisateur (référence est faite à la « république de réflexes » chez l'oursin), la notion de recherche indirecte. S'agissant de cette dernière notion, il se livre à un long commentaire de ce qu'il estime être le parti pris « intuitif» ou « visuel» (anschaulich) des schémas d'Uexküll. La biologie selon Uexküll, observe-t-il, n'établit pas des causes profondes, elle montre des fonctionnements. Elle est anschaulich - visible, intuitionnelle, macroscopique ${ }^{39}$.

Et Jennings d'exprimer ses réserves à l'égard des fictions que sont, selon lui, les schémas d'Uexküll, et son idée, formulée parfois abruptement, que l'Anschauung serait la caractéristique distinctive de la biologie. Il traduit un passage des Studien über den Tonus :

Only the anschaulich structural diagram, not proving, but showing the unified working together of different factors, is adequate to the requirement of bringing the life processes together into an intelligible unity without omitting life itself ${ }^{40}$. 
Et il poursuit :

[...] it would be extremely helpful if the author would at least segregate carefully his verifiable, experimental, results from his fictitious schema, if he finds that he cannot bring himself to totally abandon the latter.

This demand for "Anschaulichkeit" rather than verifiableness in a scientific account is what has led to an apparent opposition between v. Uexküll's work and that of some others.

[...] We shall be interested in the plan of the organism so far as it is verifiable; and to work out the verifiable plan we shall be forced to consider the actual forces, materials and arrangements, not fictitious ones. (Jennings 1909 : 329-335)

On comprend la perplexité du commentateur, et où se trouve le malentendu. Les propos d'Uexküll ne sont intelligibles que si on les articule sur les distinctions kantiennes : pour Uexküll, la physiologie est de l'ordre des causes démontrables, la biologie de l'ordre de la Planmäßigkeit, elle fait appel à l'usage régulateur de la raison. L'esthétique goethéenne n'est sans doute pas loin non plus.

Uexküll et les spécialistes de la Verhaltensforschung cheminaient donc de concert, mais sans partager certains principes structurants. Une bonne illustration en est fournie par un chercheur légèrement postérieur à Uexküll, Edward Chase Tolman (1886-1959), auteur d'un recueil d'articles ensuite regroupés sous le titre programmatique de Purposive behavior (Tolman 1932). Sans conserver l'idée uexküllienne d'un Bauplan, Tolman conçoit le milieu comme une organisation spécifique et orientée, purposive et meaningful, dans lequel le comportement est régulé par des fins objectivement déterminables. Il définit donc son béhaviorisme comme un « finalisme » (purposivism) $)^{41}$.

Such a purpose is quite an objective and purely behavioristic affair. It is a descriptive feature immanent in the character of the behavior qua behavior. It is not a mentalistic entity supposed to exist parallel to, and to run alongside of, the behavior. It is out there in the behavior; of its descriptive warp and woof. (Tolman 1951 [1926] : 51, souligné dans le texte)

On retrouve chez lui la thèse que seuls certains traits sont sélectionnés et que ce qui importe n'est pas la qualité elle-même d'un objet, mais la fonction de cette qualité - la couleur d'une pomme, par exemple, peut servir de signe distinctif à une ménagère qui fouille dans le bac de son congélateur. «Les traits distinctifs [discrimination features], écrit-il, qu'ils soient primaires ou secondaires, restent toujours uniquement des traits distinctifs » (ibid. : 57). Quelle interaction, quel intercourse, un sujet entretient-il avec son environnement? demande Tolman, qui prolonge la distinction uexküllienne entre signes de réception (Merkzeichen) et signes d'effection (Wirkzeichen), en différenciant entre les traits distinctifs (discrimination features) et les « traits de manipulation » (manipulation features). Les premiers valant signes distinctifs pour les suivants, tout comme chez Uexküll. Quel rapport puis-je avoir avec une chaise ou un bureau ? Ce rapport dépend de la conformation de l'objet et de celle de son utilisateur. " Un seul et même objet

41 Le mot finalisme est ici une traduction par défaut, qui ne rend qu'imparfaitement ce que Tolman entend par purposivism, car ce « finalisme » n'implique naturellement aucune finalité transcendante. Voir du reste la citation qui suit. Le lecteur peut aussi se reporter à ce qui est dit de Ziel (vs. Zweck) en deuxième partie. 
environnemental offrira [afford] à un animal pourvu de mains des manipulanda très différents de ceux qu'il peut offrir et offrira à un animal qui ne possède qu'une gueule » (1951 [1933] : 77-93).

A behavior-object results from a behavior-cue or a group of behavior-cues which, because of a particular behavior situation, possesses for the organization in question a specific behavior-meaning. For example, we present an ordinary western European with a chair, it produces in him, because of the structure of his sense-organs and as a result of its color, shape etc., certain specific behaviorcues. (Tolman $1922: 50$ )

Uexküll observe quant à lui :

En ce qui concerne sa signification, en tant qu'opportunité-d'assise se dressant audessus du sol, la chaise consiste en de purs ponts vers divers contrepoints. La surface d'assise, le dossier et les accoudoirs trouvent leur contrepoint dans le corps humain, vers lesquels ils forment des ponts, pendant que les pieds de chaise forment des ponts manifestes vers le contrepoint qu'est le sol. (Uexküll 1956 [1934] : 146, ma traduction)

On trouve même chez les deux auteurs des analogies identiques, telle celle de l'araignée pour figurer le rapport d'un vivant à son umwelt :

Chaque sujet tisse ses relations vers certaines propriétés des choses comme les fils d'une araignée et les entrelace jusqu'à en faire une toile solide, qui porte son être. (ibid. : 31, ma traduction)

We may liken the environment to a multidimensional spider's web radiating out from the behaving organism in many directions. The far ends of the threads terminate in final to-be-sought-for quiescences, or final to-be-avoided disturbances. Environmental objects and situations are responded to and cognized only in their character of providing bridges or routes along these threads. (Tolman 1951 [1926] : 52-53)

Uexküll (ibid. : 120-121) évoque la parfaite adéquation - le tuning dirait Tolman - de la toile et de la mouche. La toile, dit-il, est une image (Abbild) de la proie à venir, dont elle fournit une représentation négative. Dans l'univers spécifique de l'araignée, elle "représente un opérateur qui exploite la signification [Bedeutungsverwerter] du porteur de signification [Bedeutungsträger] "proie" ». Il y a, conclut-il, « accord » (musical : Einklang) entre la toile et la mouche. La toile est fliegenhaft, accordée au vol. La similitude entre les propos des deux auteurs est sensible quant aux affordances présentées par les objets, ainsi pourvus d'un meaning, d'une Bedeutung. Toutefois, outre la place qu'il accorde à la métaphore musicale ${ }^{42}$, Uexküll introduit, avec des notions comme Abbild et Vertretung, une sémiotique absente sous cette forme chez Tolman ${ }^{43}$. Ceci va nous conduire à quelques observations en guise de conclusion.

42 Plus sensible dans les textes destinés à un public large que dans TB, le registre goethéen fournit un métalangage commode. Et de nouveau la métalangue se cherche entre visée réaliste et fonction de support. On rétorquera que c'est toujours le cas, mais la biologie telle qu'Uexküll la comprend accentue cette tension.

43 Ceci ne signifie pas, bien entendu, que la perspective de Tolman soit a-sémiotique comme chez Loeb. Mais même lorsque son métalangage est explicitement sémiotique (par exemple dans Tolman 1960 [1932] : 


\section{COMMENT FIGURER UNE INCONNUE ?}

En bref, outre la notion d'umwelt, le « béhaviorisme » d'Uexküll présente deux singularités : le recours à un outillage d'inspiration kantienne et l'invocation d'un référentiel sémiotique absent, du moins sous cette forme, des autres béhaviorismes. Et ceci nous ramène à notre question initiale : Quelle est l'utilité, ou la fonction, d'un tel référentiel là où le purposivism d'un Tolman se satisfait d'identifier des affordances?

Des critiques vinrent aussi de l'autre bord, des néo-kantiens eux-mêmes. Collègue d'Uexküll à Hambourg, anti-darwinien comme lui, récusant toute forme de physicalisme, Cassirer reproche à Uexküll d'assimiler signe et symbole dans un usage indifférencié de la notion de Bedeutung, et il critique également des schémas, qui ne fournissent selon lui que des représentations spatialisées, incapables de rendre compte de la représentation abstraite à laquelle le langage humain (le symbole) permet d'accéder ${ }^{44}$. Et il faut bien reconnaitre que la sémiotique d'Uexküll ressemble davantage à une théorie des affordances traduite en formules goethéennes qu'à une théorie de la signification. - Quand Uexküll étend la notion de Ton («ton» en musique) à la tonalité sémantique des objets présents dans l'umwelt d'un sujet, il est question de points, de contrepoints et d'harmonie. Le métalangage est tantôt musical (le ton), tantôt visuel (la forme), mais une terminologie plus sobre identifiera la correspondance entre un élément positif et un élément négatif : point/contrepoint, sujet/umwelt, mouche/toile, etc., et abandonnera tout le surplus à la langue de transcription. Le cercle fonctionnel exprime en revanche un peu plus qu'une homéostase, puisque le passage du signe de perception (Merkzeichen) au signe d'effection (Wirkzeichen) correspond simultanément à une inversion de polarité ${ }^{45}$. On peut donc attribuer à Bedeutung et Zeichen une signification minimale.

Toutefois Uexküll recourt aussi à d'autres termes : Bild ou Abbild (la toile est une image [Abbild] négative de la mouche), et à un mot différent: Vertretung ${ }^{46}$. Ce mot correspond à la thèse, constamment reprise et reformulée ${ }^{47}$, que les êtres vivants, de la cellule à l'organisme complexe, ne sont jamais en contact direct avec des réalités objectives de leur environnement, mais uniquement avec des

135-160), celui-ci est sous-tendu d'une conception indicielle et associative d'un signe. (Le fait qu'il y parle de sign-gestalt risque d'induire en erreur.) En tout état de cause, on n'y trouve rien, à ma connaissance, qui corresponde à la notion uexküllienne d'Abbild, laquelle s'apparente à une analogie photographique ou mathématique, ni à celle de Vertretung, que nous allons aborder dans un instant.

44 Alors que Bedeutung chez Uexküll désigne «ce qui fait sens pour un sujet » (un siège si je souhaite m'asseoir par exemple), la sémiotique de Cassirer est au contraire logocentrée. Sur la lecture d'Uexküll par Cassirer : Chien 2006, Van Heusden 2001.

45 Ce mécanisme est d'application très générale. Par exemple, si je souhaite m'asseoir, la chaise est tour à tour objet perceptif (polarité positive) puis l'espace qui m'accueille lorsque je m'y assois (inversion de polarité).

46 Vertreten signifie « représenter », mais au sens de délégation, comme un élu est censé « représenter » un groupe de personnes par exemple. En revanche, pour désigner ce "représentant par délégation », Uexküll emploie préférentiellement le mot Repräsentant, qui a exactement ce sens, plutôt que Vertreter, qui est plus courant.

47 Uexküll 1909 : 97-98 ; TB : $175 ; 1956$ [1934] : 111 ; etc. 
réalités transformées (des représentants) et que cette transformation est spécifique. (Si je reçois un coup sur le globe oculaire, cela déclenchera des sensations lumineuses !) Cette conception apparaît dès les travaux sur le tonus musculaire, qui postulent que le centre nerveux doit être informé de l'état des muscles et pouvoir intervenir en retour sur les excitations musculaires, ce qui implique la présence d'un organe interface, le représentant, qui diffèrera selon l'espèce. Voici un schéma simplifié de la relation centre/périphérie (1905c : 51) :

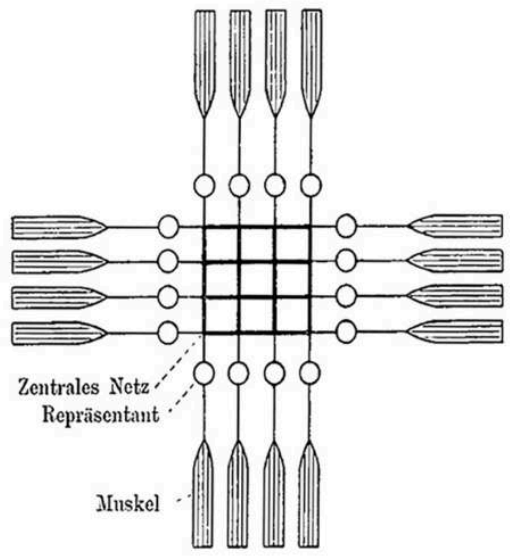

Que nous dit ce schéma? - Que l'excitation n'est pas l'influx nerveux et qu'entre l'excitation mécanique périphérique et ce qui a lieu dans le réseau central, l'information (ou «tonus ») ne circule donc qu'à condition d'être transformée. À l'interface, à proximité du centre, le «représentant» traduit l'excitation. Nous avons vu que le tonus est un concept frontière entre fonction et physiologie. Ce schéma repose sur le même paradoxe ${ }^{48}$, en reliant dans un seul espace figuratif des données anatomiques (les muscles, les nerfs) et fonctionnelles (le système central et les représentants). Ceci esquisse une réponse à notre question : tout comme le représentant est donc un artefact figuratif pour indiquer une transformation, le métalangage sémiotique permet, à un niveau plus général, d'articuler physiologie et fonction pour un coût explicatif minimal : ce qui n'est pas descriptible dans le métalangage de la chimie ou de la physiologie est dénommé dans le métalangage de la sémiotique. Ce métalangage chez Uexküll se révèle en outre plurivoque, englobant le signe comme signal (Merkmal), le signe comme image (Abbild) et le signe comme transformateur (Repräsentant). Trois valeurs dépourvues de véritable foyer commun, car adossées à deux modules explicatifs distincts : signal et image sont explicitement reliées à la Planmäßigkeit, mais signal et représentant sont par ailleurs des désignateurs vides, qui permettent de noter des inconnues.

La «sémiotique » d'Uexküll ne correspond par conséquent ni à un concept homogène, ni à un programme unique. Parler de « biosémiotique » risque même de faire illusion puisque, dans la pratique, il s'agit moins de la sémioticité 
supposée des objets que du choix d'un métalangage ${ }^{49}$, dont il est par conséquent préférable de distinguer les deux usages, qu'on appellera par commodité « interne » et « externe ». Dans son usage interne, le métalangage sémiotique décrit des formes de sémiosis (Merkmal, Abbild...). L'usage externe consiste, quant à lui, à solliciter ce métalangage en un point donné d'un schème explicatif, lequel n'est pas en soi sémiotique. C'est ce qui se passe avec la notion de représentant, qui note une inconnue dans un schéma du métabolisme. Si l'un des traits du vivant est la séparation entre un espace intérieur et un espace extérieur (par une membrane cytoplasmique, par la peau, etc. ${ }^{50}$, alors ce qui est dedans (e.g. le tonus) ne peut être de même nature que ce qui est dehors (e.g. une irritation quelconque). La notion de représentant circonscrit cette différenciation. « J'ai proposé [...] d'appeler les centres musculaires des "représentants" », écrit Uexküll.

Ce nom nous conduit immédiatement à la compréhension de la fonction fondamentale du système nerveux central. Le système nerveux central ne peut faire autre chose qu'ordonner des excitations. S'il doit régir le corps tout entier par cette seule capacité, alors cela ne peut se produire que si, d'une part, toutes les irritations du monde extérieur sont converties en excitations, et si, d'autre part, tous les mouvements corporels peuvent être activés par des excitations. [...] Il doit donc y avoir un organe nerveux qui soit influencé par l'état des muscles et qui puisse quant à lui agir sur les excitations. Cet organe, ce sont les représentants. (Uexküll 1909 : 97-98)

Uexküll use d'un artefact descriptif similaire pour distinguer umwelt et environnement matériel (Umgebung) : si chaque umwelt est spécifique et si les umwelten ne se fondent jamais dans une Umgebung commune, c'est que chaque espèce répond, non à des stimuli matériels, mais à certains traits pertinents, des Merkmale, autrement dit des « signes ». Peu importe finalement qu'assimiler la sélectivité à une sémiotique confine à une pétition de principe. Le signe (Zeichen) permet, ici également, de localiser le surplus propre au vivant.

Air du temps sans doute, ces Merkmale sont très semblables à ceux de Bühler, que ce dernier définit dans sa Sprachtheorie par le principe de "pertinence abstractive » (abstraktive Relevanz) ${ }^{51}$. Mais la similitude ne s'arrête pas là, car le couple Umgebung/Umwelt a un pendant logique dans la Sprachtheorie. La thèse centrale d'Uexküll est que le vivant et l'inerte ne s'insèrent pas dans le monde environnant de la même manière. Alors que le lien entre le vent et la forme du nuage est purement mécanique, explique-t-il (1909: 114), il n'en va pas de même de l'ombelle du pissenlit ou de la samare de l'érable. Dans ce cas, ce n'est pas le vent qui cause la forme, «les formes sont ajustées au facteur de signification

49 Une affaire de méthodologie donc, et non d'ontologie. Le cas d'Uexküll est involontairement prophylactique, en interpellant tout sémioticien qui s'interrogerait sur la nature des signes avant de se demander dans quelle mesure l'idée même de signe est un artefact méthodologique.

50 C'est ce qu'indique le titre Innen- und Aussenwelt der Tiere, «monde interne et monde externe des animaux ».

51 La Sprachtheorie de Bühler est parue en 1934, soit la même année que l'édition originale de Streifzüge. Pour ce qui va suivre, et notamment les notions de champ et de pertinence abstractive, voir le « glossaire » qui accompagne la traduction française (Bühler 2009 [1934] : 617-667). 
[Bedeutungsfaktor] "vent" »( ibid.). Or cette distinction correspond à deux « valeurs de champ » (Feldwerte) distinguées par Bühler. En langage bühlérien, on dirait en effet que le rapport du nuage à son champ de vent est « symphysique » (purement matériel), mais que celui de la samare est «sympratique» car, à la différence du nuage, la samare est syntaxiquement intégrée dans son champ. En langage uexküllien, le nuage n'a qu'une Umgebung tandis que la samare a une umwelt.

Qu'est-ce qui distingue dans ce cas les métalangages d'Uexküll et de Bühler? Ce n'est pas leur contenu. Ils se distinguent clairement sur deux aspects corrélés. Premièrement, ce qu'Uexküll exprime par des métaphores goethéennes illustrées d'exemples, Bühler le formule dans un métalangage général et axiomatisé, en l'occurrence la théorie des champs. Deuxièmement et a contrario, on chercherait en vain chez lui quelque chose qui correspondrait à la distinction uexküllienne entre l'inerte et le vivant. La sémiotique chez Bühler n'a pas de fonction externe. La situation est inverse chez Uexküll : dépourvue de modèle intégré, elle sert d'artefact de jonction pour circonscrire les propriétés du vivant.

Que l'époque ait buté sur la difficulté à penser le vivant est une évidence ${ }^{52}$, et le procédé n'a rien de scandaleux. Si la chimie n'est pas en mesure de rejoindre le vivant, alors il faut bien combler les intervalles manquants en leur attribuant une désignation conventionnelle, et les intégrer comme autant d'inconnues dans le calcul, ainsi que le montre le schéma ci-dessus. Pour l'historien, le problème n'est donc pas tant la méthode elle-même que la conjoncture qui, agrégeant des composants très divers, conduit à tels et tels métalangages, à telles et telles conventions d'écriture. Il ressort de ce qui précède qu'Uexküll a forgé les siennes en greffant des notions sémiotiques, avec leur éventuel apport technique ${ }^{53}$, sur un couple central de la Critique de la faculté de juger.

\section{BIBLIOGRAPHIE}

Arstila, Valterri \& Dan E. Loyd, éd. 2014. Subjective Time: The Philosophy, Psychology, and Neuroscience of Temporality. Cambridge, Mass. : MIT Press.

Baer, Karl Ernst von. 1864. Welche Auffassung der lebenden Natur ist die richtige? Und wie ist diese Auffassung auf die Entomologie anzuwenden? Reden gehalten in wissenschaftlichen Versammlungen und kleinere Aufsätze vermischten Inhalts, vol. 1, dir. par Karl Ernst von Baer. St. Petersbourg : H. Schmitzdor. 237-284.

Beer, Theodor, Albrecht Bethe \& Jakob von Uexküll. 1899. Vorschläge zu einer objectivierenden Nomenklatur in der Physiologie des Nervensystems. Biologisches Centralblatt 19 : 517-521.

Bertalanffy, Ludwig von. 1932-1942. Theoretische Biologie. 2 vol. Berlin : Gebrüder Borntraeger.

Bertalanffy, Ludwig von. 2006 [1969]. General System Theory: Foundations, Development, Applications. New York : Braziller.

52 Les réflexions de Claude Bernard en fournissent une belle illustration, avec des solutions somme toute assez voisines de celles proposées par Uexküll. Sur le vitalisme de Claude Bernard : Malaterre 2007.

53 Je n'ai pu évoquer que très rapidement la discrétisation des grandeurs continues. Uexküll la formule en termes sémiotiques (voir notes 21 et 39 ). 
Bühler, Karl. 2009 [1934]. Théorie du langage. La fonction représentationnelle. Marseille: Agone [publication originale: Sprachtheorie. Die Darstellungsfunktion der Sprache. Iéna : Gustav Fischer. 1934].

Chien, Jui-Pi. 2004. Schema as Both the Key to and the Puzzle of Life: Reflections on the Uexküllian Crux. Sign Systems Studies 32(1/2) : 187-208.

Chien, Jui-Pi. 2006. Of Animals and Men: A Study of Umwelt in Uexküll, Cassirer and Heidegger. Concentric: Literary and Cultural Studies 32(1) : 57-79.

Driesch, Hans. 1892. Entwicklungsmechanische Studien. I: Der Wert der beiden ersten Furchungszellen in der Echinodermententwicklung. Experimentelle Erzeugung von Teil- und Doppelbildungen. II: Über die Beziehungen des Lichtes zur ersten Etappe der thierischen Formbildung. Zeitschrift für wissenschaftliche Zoologie 53 : 160-184.

Driesch, Hans. 1919. Der Begriff der organischen Form. Berlin : Gebrüder Borntraeger.

Ehrenberg, Rudolf. 1923. Theoretische Biologie. Vom Standpunkt der Irreversibilität des Elementaren Lebensvorganges. Berlin : Julius Springer.

Fontanille, Jacques. 2019. La sémiotique des mondes vivants. Du signe à l'interaction, de la téléologie à la structure. Actes sémiotiques 122 [www.doi.org/10.25965/ as.6233, consulté le 13/09/2021].

Jennings, Herbert S. 1906. Behavior of the Lower Organisms. Londres \& New York : Macmillan \& Co.

Jennings, Herbert S. 1909. The Work of J. von Uexkuell on the Physiology of Movements and Behavior. Journal of Comparative Neurology and Psychology $19: 313-336$.

Kant, Immanuel. 1922 [1790]. Kritik der Urteilskraft. Leipzig : Felix Meiner.

Koffka, Kurt. 1935. Principles of Gestalt Psychology. New York : Harcourt, Brace.

Kull, Kalevi. 2001. Jakob von Uexküll: An introduction. Semiotica 134(1/4) : 1-59.

Kull, Kalevi. 2015. Semiosis Stems from Logical Incompatibility in Organic Nature: Why Biophysics Does Not See Meaning, While Biosemiotics Does. Progress in Biophysics and Molecular Biology 119(3) : 616-621.

Lattal, Kennon A. \& Alexandra Rutherford. 2013. John B. Watson's Behaviorist Manifesto at 100. El manifiesto conductista de John B. Watson a sus 100 años. Mexican Journal of Behavior Analysis. Revista Mexicana de Análisis de la Conducta 39(2) : 1-9.

Malaterre, Christophe. 2007. Le «néo-vitalisme » au XIX siècle : une seconde école française de l'émergence ? Bulletin d'histoire et d'épistémologie des sciences de la vie $14(1): 25-44$.

Merleau-Ponty, Maurice. 2001. Psychologie et pédagogie de l'enfant: Cours de Sorbonne 1949-1952. Lagrasse : Verdier.

Mildenberger, Florian. 2006. The Beer/Bethe/Uexküll Paper (1899) and Misinterpretations Surrounding "Vitalistic Behaviorism". History and Philosophy of the Life Sciences 28(2) : 175-189.

Pöppel, Ernst. 2004. Lost in Time: A Historical Frame, Elementary Processing Units and the 3-Second Window. Acta Neurobiologicae Experimentalis 64(3) : 295-301. 
Reinke, Johannes. 1901. Einleitung in die theoretische Biologie. Berlin : Gebrüder Paetel.

Rothschuh, Karl E. 1936. Theoretische Biologie und Medizin. Zur biologischen Grundlegung und Wissenschaftstheorie der Medizin. Berlin : Junker und Dünnhaupt.

Russell, Edward S. 1934. The Study of Behaviour. Nature 1 : 835-839.

Russell, Edward S. 1938 [1934]. The Behaviour of Animals. An Introduction to its Study. Londres : Edward Arnold \& Co.

Samain, Didier. 2018a. Bühler, la Gestalt et le béhaviorisme. Karl Bühler, une théorie du langage redécouverte, dir. par Tomáš Hoskovec et al. Prague : OPS éditions (Travaux du Cercle linguistique de Prague, 7). 267-295.

Samain, Didier. 2018b. L'écologie béhavioriste des signes au regard du paradigme saussurien. Travaux des colloques. Le Cours de linguistique générale, 1916-2016. L'émergence, le devenir, dir. par Daniele Gambarara \& Fabienne Reboul. Genève : Cercle Ferdinand de Saussure. 100-110.

Samain, Didier. 2020. Technique et fiction chez Trần Đức Thảo. Qu'est-ce qu'un contexte d'activité ? Histoire Épistémologie Langage 42(2) : 17-34.

Schloegel, Judy J. \& Henning Schmidgen. 2002. General Physiology, Experimental Psychology, and Evolutionism. Unicellular Organisms as Objects of Psychophysiological Research, 1877-1918. Isis 93(4) : 614-645.

Tolman, Edward C. 1922. A New Formula for Behaviorism. Psychological Review 29(1) : 44-53.

Tolman, Edward C. 1951 [1926]. A Behavioristic Theory of Ideas. Behavior and Psychological Man, par Edward C. Tolman. Berkeley \& Los Angeles : University of California Press. Londres : Cambridge University Press. 48-62 [publication originale dans Psychological Review 33(5) : 352-369. 1926].

Tolman, Edward C. 1951 [1933]. Gestalt and Sign-Gestalt. Behavior and Psychological Man, par Edward C. Tolman. Berkeley \& Los Angeles : University of California Press. Londres : Cambridge University Press. 48-62 [publication originale dans Psychological Review 40(5) : 391-411. 1933].

Tolman, Edward C. 1951. Behavior and Psychological Man. Berkeley \& Los Angeles : University of California Press. Londres : Cambridge University Press.

Tolman, Edward C. 1960 [1932]. Purposive Behavior in Animals and Men. New York \& Londres : Century.

Uexküll, Jakob von. 1896. Zur Muskel- und Nervenphysiologie von Sipunculus nudus. Zeitschrift für Biologie 33 : 1-27.

Uexküll, Jakob von. 1900a. Die Physiologie des Seeigelstachels. Zeitschrift für Biologie 39 : 73-112.

Uexküll, Jakob von. 1900b. Die Wirkung von Licht und Schatten auf die Seeigel. Zeitschift für Biologie 40 : 447-476.

Uexküll, Jakob von. 1902. Psychologie und Biologie in ihrer Stellung zur Tierseele. Ergebnisse der Physiologie. Biophysik und Psychophysik 1(2) : 212-233.

Uexküll, Jakob von. 1903. Studien über den Tonus I. Der biologische Bauplan von Sipunculus nudus. Zeitschrift für Biologie 26 : 269-344. 
Uexküll, Jakob von. 1905a. Studien über den Tonus II. Die Bewegungen der Schlangensterne. Zeitschrift für Biologie 28 : 1-37.

Uexküll, Jakob von. 1905b. Studien über den Tonus III. Die Blutegel. Zeitschrift für Biologie 28 : 372-402.

Uexküll, Jakob von. 1905c. Leitfaden in das Studium der experimentellen Biologie der Wassertiere. Wiesbaden : J. F. Bergmann.

Uexküll, Jakob von. 1907a. Studien über den Tonus IV. Die Herzigel. Zeitschrift für Biologie 31 : 307-332.

Uexküll, Jakob von. 1907b. Die Umrisse einer kommenden Weltanschauung. Die neue Rundschau 18 : 641-666.

Uexküll, Jakob von. 1908. Studien über den Tonus V. Die Libellen. Zeitschrift für Biologie 32 : 168-202.

Uexküll, Jakob von. 1909. Umwelt und Innenwelt der Tiere. Berlin : Julius Springer.

Uexküll, Jakob von. 1912. Studien über den Tonus VI. Die Pilgermuschel. Zeitschrift für Biologie 40 : 305-332.

Uexküll, Jakob von. 1913. Der heutige Stand der Biologie in Amerika. Die Naturwissenschaften 34 : 801-805.

Uexküll, Jakob von. 1920. Theoretische Biologie. Berlin : Gebrüder Paetel.

Uexküll, Jakob von. 1926 [1920]. Theoretical Biology. Londres : Kegan Paul, Trench, Trubner \& Co. [publication originale : Theoretische Biologie. Berlin : Gebrüder Paetel. 1920].

Uexküll, Jakob von. 1935. Die Bedeutung der Umweltforschung für die Erkenntnis des Lebens. Zeitschrift für die gesamte Naturwissenschaft 1 : 257-272.

Uexküll, Jacob von. 1965 [1934]. Mondes animaux et monde humain. Suivi de Théorie de la signification. Paris : Gonthier [publication originale : Streifzüge durch die Umwelten von Tieren und Menschen. Bedeutungslehre. Hambourg: Rowohlt. 1956 (1934)].

Uexküll, Jakob von. 1973 [1928]. Theoretische Biologie. $2^{\mathrm{e}}$ éd. entièrement révisée. Frankfort-sur-le-Main : Suhrkamp.

Uexküll, Jakob von \& Georg Kriszat. 1956 [1934]. Streifzüge durch die Umwelten von Tieren und Menschen. Bedeutungslehre. Hambourg : Rowolt.

Van Heusden, Barend. 2001. Jakob von Uexküll and Ernst Cassirer. Semiotica $134(1 / 4): 275-292$.

Watson, Johan B. 1913. Psychology as the Behaviorist Views It. Psychological Review 20 : 158-177.

Weismann, August. 1892. Das Keimplasma. Eine Theorie der Vererbung. Iéna: Gustav Fischer. 